\title{
'UNA MYGA YMATGE EN PAPER, DE PLOMA, DE MÀ DE JOHANNES'. LA FUGITIVA SOMBRA DE VAN EYCK EN LA CORONA DE ARAGÓN A MEDIADOS DEL SIGLO XV
}

\author{
EnCARNA MONTERo TORTAJADA \\ Universitat de València \\ Encarna.Montero@uv.es
}

El artículo analiza la mención de un dibujo a pluma atribuido a Van Eyck en un inventario post-mortem fechado en 1452, y realizado en Valencia. Los bienes del elenco pertenecieron a Andreu Garcia, presbítero beneficiado en la catedral de la ciudad bien conocido por la historiografía en virtud de sus importantes vínculos con los artistas más señalados que trabajaron en la urbe durante la primera mitad del siglo XV. La noticia da pie a una nueva revisión del cada vez más improbable paso de Van Eyck por los territorios de la Corona de Aragón, así como de la huella temprana de su pintura en estos lugares, habida cuenta de que, como revela el documento, el primer propietario del dibujo fue Berenguer Mateu, pintor hermano de Jaume Mateu, uno de los mejores representantes de la segunda generación de pintores del Gótico Internacional en la capital del Reino.

Palabras clave: Van Eyck; Valencia; arte medieval; dibujo; mediados del siglo XV.

'UNA MYGA YMATGE EN PAPER, DE PLOMA, DE MÀ DE JOHANNES'. THE FLEETING SHADOW OF VAN EYCK IN ARAGÓN DURING THE MID-15TH CENTURY

This paper analyses the mention of a quill drawing attributed to Van Eyck in a Valencian postmortem inventory, dated 1452. The properties belonged to Andreu Garcia, a priest well known in the historical record because of his connections with the most notable artists working in Valencia during the first half of the $15^{\text {th }}$ century. This reference compels a new examination of the ever more improbable presence of Van Eyck in Aragonese territories. It also offers a new insight into the early imprint of his paintings in these lands, given that the first owner of the drawing was the painter Berenguer Mateu (Jaume Mateu's brother, likewise a prominent Valencian painter).

Key words: Van Eyck; Valencia; Medieval Art; drawing; mid- $15^{\text {th }}$ century.

En noviembre de 1452 expira en Valencia el presbítero Andreu Garcia, prohombre de la Iglesia nacido en el seno de la oligarquía urbana, fuertemente vinculado a la orden cartuja y al franciscanismo espiritual. Además de acumular varios beneficios eclesiásticos, es sabido que actuó como mediador en la concreción de buen número de encargos que implicaron a lo más gra- 
nado de la menestralía artística que trabajó en Valencia entre 1420 y 1452. En su testamento, recibido por el notario Ambrosi Alegret en 1450, Garcia dispone que se fabriquen varios ornamentos litúrgicos y que se construya una capilla en el claustro de la cartuja de Valldecrist, monasterio donde dispone ser enterrado en hábito de monje. Sin embargo, el inventario de sus bienes es el documento que pone al descubierto el interés del presbítero por la práctica del dibujo, y la red casi clientelar que estableció con varios artistas de renombre ${ }^{1}$. Siendo interesante la figura de Garcia sólo por esto, a todo lo anterior debe sumarse una entrada de este listado de enseres tan intrigante como sugestiva:

Ítem, un libre de forma de full ligat e un paper ligat ab aquell de mà de mossèn Andreu Guarcia és scrit: "Aquest libre de pintura e una miga ymatge en paper, de ploma de mà de Johannes, e les quatre taules ligat, ab les testes pintades, me prestà en Berenguer Mateu, $[\ldots]$ pintor, jermà d'en Jacme Matheu. És veritat que yo li prestí sis florins, et cetera, e n'e cobrats los tres florins et cetera, és intenció mia que lo dit libre sia tornat a son germà o als seus de qui és [...] et cetera" ${ }^{2}$

Siempre que se inicia el comentario de un fragmento transcrito es importante establecer con claridad qué es lo que se puede deducir de su lectura: en este caso se está inventariando un libro "de forma de full ligat" al que hay atado un papel. Éste resulta ser una nota aclaratoria de Garcia, en la que señala que ese libro de pintura, una media imagen en papel dibujada a pluma "de mà de Johannes", y cuatro tablas unidas en las que hay cabezas pintadas son propiedad del pintor Berenguer Mateu, hermano del también pintor Jaume Mateu (uno de los más eximios representantes de la segunda generación del Gótico Internacional en Valencia), quien los dejó como fianza por un préstamo de seis florines, de los que había devuelto ya tres en el momento en el que Garcia redactó la nota. Es necesario subrayar que el inventario da cuenta del libro y de la nota aclaratoria, pero no del dibujo ni de las tablillas. Por otra parte, Andreu Garcia estipula que el libro se devuelva al hermano de Berenguer Mateu o a los suyos, aunque nada dice del dibujo a pluma ni de las tablas, que tal vez tuviera intención de retener hasta el cobro del montante total de la cantidad prestada.

Así, hay tres pertenencias de Mateu que se encuentran temporalmente en poder de Garcia: un libro de pintura, cuatro tablas unidas con cabezas pintadas, y el dibujo a pluma. En otros estudios ya se ha reflexionado por extenso sobre el libro de pintura ${ }^{3}$ y el particular significado de las tablas -de ésas, y de otras muchas que aparecieron en el inventario- en la transmisión del conocimiento artístico ${ }^{4}$. Corresponde ahora examinar el dibujo a pluma, vórtice de muchas consideraciones por estar relacionado con una sola palabra: "Johannes". En primer lugar, es necesario establecer a qué artista se podría referir la documentación en 1452 con ese apelativo. Si fuera un pintor autóctono, casi sin duda en su nota Garcia hubiera aludido a él en vernáculo como "Joan", agregando algún dato más que precisase su identidad. Debió tratarse, pues, de un artífice extranjero, y bien conocido, por cuanto es el "Juan" por antonomasia. Además, el presbítero se esfuerza en aclarar que se trata de una obra autógrafa, lo que también resulta bien explícito del aprecio que se tiene por su autor. En Valencia, a mediados del siglo XV, estas valoraciones sólo se podrían sostener atribuyéndolas a Jan van Eyck. La aparición del dibujo concuerda cronológicamente con otras dos obras suyas que pasan por la ciudad en esos mismos años, a las que justo la documentación se refiere de manera idéntica: el San Jorge que embarca en el puerto de Valencia con destino a Nápoles en 1444, comprado por Alfonso V, y la Estigmatización de San Francisco que poseía el pintor Joan

\footnotetext{
${ }^{1}$ Montero Tortajada, 2013b.

2 Archivo de Protocolos del Corpus Christi de Valencia (APCCV), nº 1107, Ambròs Alegret, 13 de noviembre de 1452.

${ }^{3}$ Un análisis del significado de éste, en Montero Tortajada, 2007.

${ }^{4}$ Sobre esta cuestión, vid. Montero Tortajada, 2013a: 221-242.
}

Arch. esp. arte, LXXXIX, 353, ENERO-MARZO 2016, 1-14 ISSN: 0004-0428, eISSN: 1988-8511, doi: 10.3989/aearte.2016.01 
Reixac ${ }^{5}$. Aun así, tan sólo es posible establecer, de momento, que en el inventario "Johannes" muy probablemente quiso decir "Jan van Eyck". Berenguer Mateu poseyó entonces un dibujo que consideró de su mano, y éste pasó en fecha indeterminada en calidad de depósito a manos de un presbítero aficionado a la pintura como era Andreu Garcia. Se está, de esta manera, ante un espectro fugaz, ante la fantasmagoría de un dibujo autógrafo de Van Eyck en manos de un eclesiástico valenciano a mediados del siglo XV, sustentada, principalmente, en dos argumentos. El primero de ellos es la noticia de otras obras de Van Eyck en la ciudad. El segundo, mucho más inaprensible, es el posible paso del pintor por la urbe en 1427 como integrante de la comitiva de una embajada de la Corte Borgoñona a la Corona de Aragón. Así, es necesario revisar qué documentos refrendan lo anterior ${ }^{6}$ para saber qué es lo que puede haber de verosímil en la atribución del inventario.

Se comenzará por el supuesto viaje de Van Eyck a la península Ibérica en 1427, posible base y explicación, caso de comprobarse algún día su existencia, de la presencia de obras del pintor en Valencia a mediados del siglo XV. Está documentado con razonable certeza el desplazamiento del artista en 1428 desde Flandes a Portugal como parte del séquito de la embajada borgoñona que tuvo como objetivo pedir la mano de la primogénita de Juan I, Isabel, para el Duque Felipe. La razón de la presencia de Van Eyck en la comitiva fue, claro está, retratar a la prometida tras el éxito de las conversaciones en las que se trató el compromiso. El pintor llevó a cabo su cometido en Avís por partida doble y remitió a Flandes un ejemplar por mar y otro por tierra. Es importante señalar que en Lisboa, donde Van Eyck estuvo del 18 de diciembre de 1428 a principios de enero de 1429, se supone que tuvo lugar un encuentro entre el pintor y Lluís Dalmau, quien tal vez formara parte de la comitiva aragonesa que acompañaba al Infante don Pedro de Portugal de vuelta a su tierra, tras haber solicitado la mano de Leonor, hermana de Alfonso V de Aragón (el Infante desembarca en Valencia en julio de 1428) ${ }^{7}$. Van Eyck sólo regresará a Flandes en diciembre del año siguiente (1429). Por eso se cree que, aunque no se le mencione explícitamente, tuvo que formar parte del séquito de la embajada del Duque de Borgoña para pretender la mano de la infanta Leonor el año anterior $(1428)^{8}$, ya que el 18 de octubre de 1427 se sabe que Van Eyck pasa por Tournai, donde es obsequiado con el vino que la ciudad suele dar a sus huéspedes distinguidos, y que algo antes, a fines de septiembre o a principios de octubre de 1427, una embajada borgoñona embarca en el Grao de Valencia, después del fracaso de la misión. El 27 de julio la comitiva habría estado en Barcelona, y en agosto habría pasado por Valencia ${ }^{9}$.

Es oportuno señalar aquí, antes de proseguir con el examen del improbable viaje de 1427, que "Johannes" es una forma latina, y el latín es la lengua en la que muy probablemente la embajada de la Corte Borgoñona se comunicase, y en la que los integrantes de la misma se presentarían a los interlocutores con los que se relacionasen durante su estancia. De todas formas, las fechas de este posible primer desplazamiento son muy tempranas, alrededor de cinco años antes de las primeras obras de

\footnotetext{
${ }^{5}$ La noticia referente al San Jorge la publicó Sanchis Sivera, 1914: 114; la última voluntad de Reixac fue exhumada por Cerveró Gomis, 1964: 93. Tanto en el libro de Cuentas de la Bailía como en el testamento se cita a "Johannes". Todos estos datos se revisarán con detenimiento en párrafos posteriores.

${ }^{6}$ Para una primera aproximación al asunto, vid. Pemán y Pemartín, 1969: 29-52 (monografía ampliamente superada, aunque válida como punto de partida, ya que cita las fuentes de las que deriva toda la bibliografía posterior); Company, 1990: 7-42; Company, 1991: vol. I, 22-26; Benito Doménech y Gómez Frechina, 2001. Vid. particularmente Cornudella, 2009-2010: 39-62, como revisión crítica apuradísima de todo lo anterior. Para un estudio reciente, vid. Fransen, 2012: $39-58$.

7 Tal y como se ha apuntado en la nota anterior, la presencia de Dalmau en Lisboa ha sido puesta seriamente en entredicho por Cornudella, 2009-2010: 48. El autor aporta más argumentos que cuestionan el viaje del pintor valenciano en la nota 44.

${ }^{8}$ Sobre la condición de cortesano de Jan van Eyck, vid. Harbison, 1991: 19-24.

9 Esta es la explicación sucinta de los hechos según Pemán y Pemartín, 1969: 29-32 (con las correcciones pertinentes tras haber leído a Paviot y a Cornudella).
}

Arch. esp. arte, LXXXIX, 353, ENERO-MARZO 2016, 1-14

ISSN: 0004-0428, eISSN: 1988-8511, doi: 10.3989/aearte.2016.01 
Van Eyck fechadas con seguridad. De este modo, la suposición más directa es pensar que el dibujo a pluma fuera un rastro de la primera etapa de su producción, aunque también pudo llegar a manos de Berenguer Mateu, que es su primer dueño documentado, más tarde, cuando la obra del flamenco ya era bien conocida y muy valorada, veinticinco años después de su eventual paso por la ciudad ${ }^{10}, \mathrm{y}$ once después de su muerte. La posesión de una pieza de "Johannes" debió ser un sello de prestigio para cualquier pintor, y más si se considera que Van Eyck era uno de los artistas predilectos del Rey. Por otra parte, en el caso - casi quimérico- de que el dibujo fuera un rastro de la presencia pasajera del pintor en la urbe, una reliquia adquirida por Mateu desde primera hora, se debería considerar cuál pudo ser la edad y cuál el prestigio profesional de éste hacia 1427, así como la huella flamenca que pudo quedar en su pintura. Por el momento, se sabe que a partir de 1423 Berenguer Mateu aparece como pintor en la documentación valenciana ${ }^{11}$, y que ya en 1432 participa en una empresa de notable envergadura como era la pintura de los muros y las bóvedas de la capilla mayor catedralicia, cobrando tanto como Miquel Alcanyís o Felip Porta ${ }^{12}$. Algunos años antes, entre 1428 y 1431, contrató tres retablos, dos de ellos para Jérica y otro para Oliva ${ }^{13}$. Así pues, cabe suponer que en 1427 debía ser un artífice prometedor. Hasta hace muy poco, la cuestión de la posible influencia directa de la pintura nórdica (de raíz eyckiana o no) en su arte era una cuestión difícil de abordar, ya que no constaba ninguna obra conservada que pudiera relacionarse de un modo u otro con su labor. Ésta, a tenor de la documentación conocida, parecía orientarse, con excepción quizás de su participación en el proyecto de la cabecera de la Seu, eminentemente hacia lo ornamental. La idea anterior debe revisarse tras la exhumación de un documento clave, dado a conocer por Joan Aliaga y Stefania Rusconi muy recientemente $^{14}$. Se trata del contrato que atribuye a Berenguer la autoría del retablo de San Jorge, conservado en Jérica (fig. 1). El acuerdo data de diciembre de 1430, y en él la cofradía del Santo encarga al pintor un mueble de trece palmos de altura por un precio de 47 florines, cantidad comparable a la que suelen cobrar otros artífices coetáneos de primera línea. La obra, restaurada entre 1998 y 2000 por el Centro Técnico de Restauración del Museo de Bellas Artes de Valencia, y expuesta desde entonces en el Museo Municipal de Jérica ${ }^{15}$, ha sido atribuida a diversas personalidades del Gótico Internacional en Valencia. Una vez conocido el autor de la pieza, Aliaga Morell y Rusconi consideran que éste muestra bien la transición desde los epígonos del estilo 1400 a la primera pintura influida por Flandes. Este temprano eco del arte septentrional se concreta, según los investigadores, en el realismo con el que está representado el caballo del Santo, en la tabla central, y en las vestiduras de la princesa. Aun así, resulta claro que, en cuanto a iconografía y composición, la obra muestra la impronta clara del retablo del Centenar de la Ploma. Ése es el modelo que gravita sobre la pieza de Jérica, y no otro. De esta forma, el alcance de la influencia nórdica en la pintura de Berenguer Mateu se convierte en un asunto tan vidrioso como interesante, ya que si el pintor poseyó un dibujo flamenco, su obra conservada apenas se muestra imantada por esta influencia.

${ }^{10}$ La bibliografía sobre Jan van Eyck es prácticamente inabarcable. Sobre el primer periodo de su carrera se remite aquí al volumen ya clásico de Panofsky, 1998 (1 ${ }^{\text {a }}$ ed. en 1953); al texto fundacional de Sterling, 1976; y a la monografía de Otto Pächt sobre los pioneros de la pintura neerlandesa [Pächt, 1994: 79-118]; así como a la colección de estudios editados por la National Gallery de Londres con el título Investigating Jan van Eyck [Foister, Jones y Cool, 2000: 183207].

${ }^{11}$ Para una revisión de la figura de Berenguer Mateu, vid. Aliaga Morell y Rusconi (en prensa). La autora desea hacer constar aquí su gratitud al Dr. Joan Aliaga, por permitirle leer amablemente el texto aún inédito de un estudio que aporta gran número de datos nuevos, los cuales modifican de forma clara la percepción de la pintura del menor de los Mateu. Cfr. también Cerveró Gomis, 1963: p. 123; 1966: 22-23; 1971: 34. Se agradece igualmente a Carme Llanes la cesión de las noticias de las que disponía sobre el pintor, tanto las publicadas como las inéditas.

12 Aliaga Morell, 1996: p. 202 (doc.49).

13 Aliaga Morell y Rusconi (en prensa).

${ }^{14}$ Aliaga Morell y Rusconi (en prensa).

15 Blackman y Ferre Puerto, 2001. 


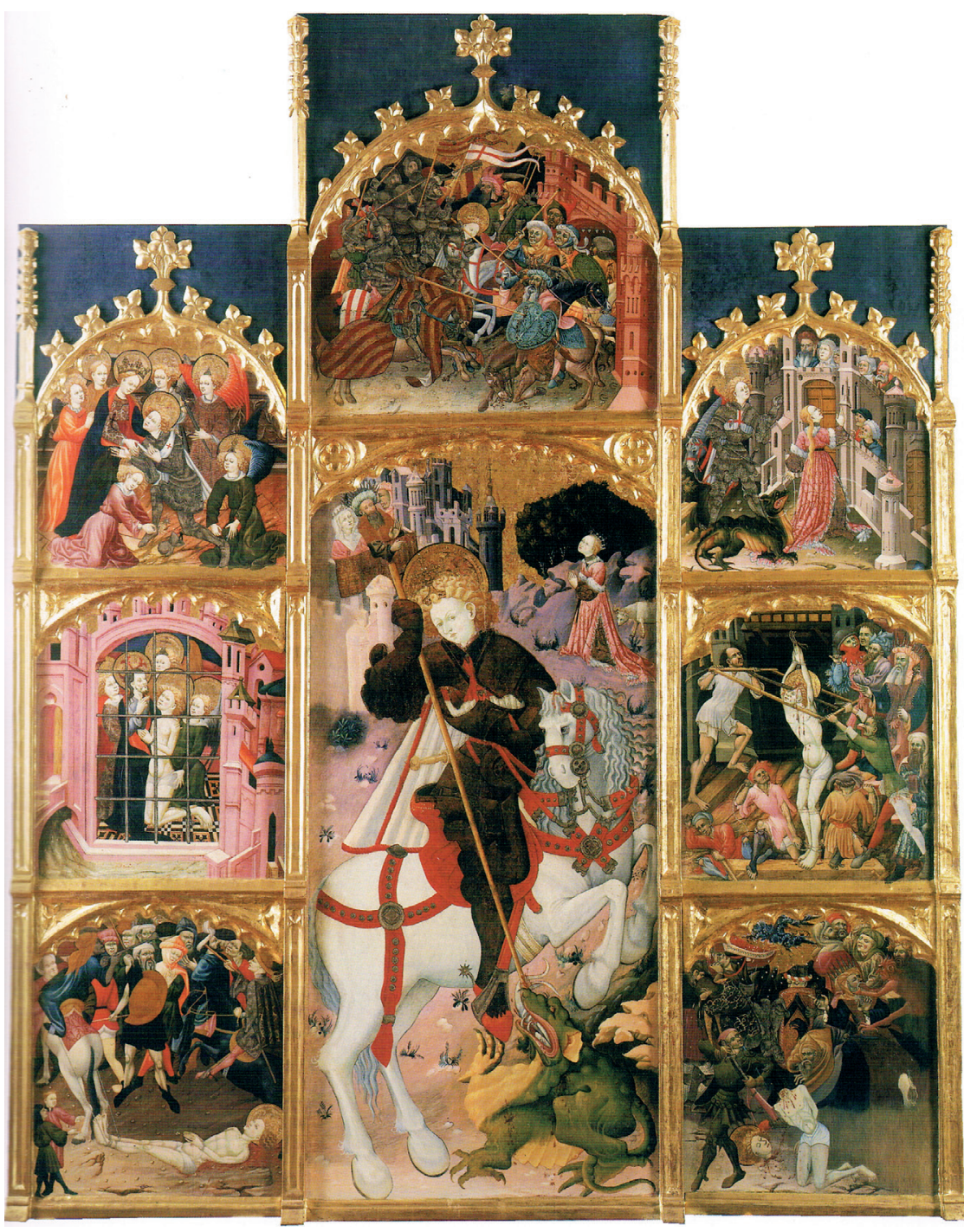

Fig. 1. Berenguer Mateu, Retablo de San Jorge (ca.1431). Museo Municipal de Jérica.

Retomando por fin las consideraciones sobre la presencia de Jan van Eyck en Aragón, a favor de ella se han inclinado estudiosos como Eduard Mira ${ }^{16}$ o Ximo Company ${ }^{17}$. Bastante más cautos al respecto se muestran Mauro Natale ${ }^{18}$, Carl Brandon Strehlke ${ }^{19}$, y Jacques Paviot ${ }^{20}$, interpretando de nuevo dos noticias publicadas por Weale ${ }^{21}$, que es de quien derivan muchos de los datos docu-

${ }^{16}$ Eduard Mira, "Del Mar del Norte al Mediterráneo. Vínculos dinásticos, rutas comerciales y relaciones artísticas", en Natale, 2001: 126.

${ }^{17}$ Company, 1990: 9-10.

${ }_{18}$ Mauro Natale, "El Mediterráneo que nos une", en Natale, 2001: 30.

${ }^{19}$ Strehlke, 1997: 55.

${ }^{20}$ Paviot, 1990: 83-93.

${ }^{21}$ Weale, 1908: XXXIII, documento 10. El trabajo de Weale lo cita Paviot (1990: 86) en su edición de 1908.

Arch. esp. arte, LXXXIX, 353, ENERO-MARZO 2016, 1-14

ISSN: 0004-0428, eISSN: 1988-8511, doi: 10.3989/aearte.2016.01 
mentales conocidos sobre Van Eyck. En concreto, Paviot demuestra mediante tres noticias que el pintor no formó parte de la expedición que viajó a Aragón: el artista recibió dos pagos y fue regalado con vino por la ciudad de Tournai entre julio y octubre de $1427^{22}$, justo en el periodo en el que la comitiva que visitó Valencia estuvo fuera de Flandes ${ }^{23}$. El autor, además, corrige la datación de este periodo, que comprende desde el 1 de junio de 1427 hasta el 15 de febrero de 1428, y no hasta el 18 de octubre del año anterior, como se venía creyendo.

Lo que se sabe respecto a la eventual presencia de Jan van Eyck en la embajada borgoñona que visitó Valencia, circunstancia que cada vez se perfila como más improbable, queda expuesto. No obstante, excluir la presencia de Van Eyck en Valencia no impide que su obra pudiera llegar a la ciudad por otras vías. Se sabe con seguridad, como anteriormente se ha apuntado, que un San Jorge del pintor que embarcó en el puerto de Valencia a bordo de la galera genovesa "Negrona"24, con destino a Nápoles, fue comprado para Alfonso V en la ciudad por dos mil sueldos en $1444^{25}$. Consta también una tabla con la Estigmatización de San Francisco "de la mà de Johannes" que Joan Reixac en su testamento de 1448 cede a Andreu Garcia mientras éste viva ${ }^{26}$, al tiempo que queda designado como tutor de los hijos del pintor. Con esto, en casa de Garcia hubieran podido entrar en contacto dos obras atribuidas a Van Eyck entre 1448 y 1452: no será así porque el presbítero fallece en esta última fecha, y Reixac no lo hace hasta 1486. A pesar de esto, la coincidencia de estas dos obras en un mismo domicilio no pasaría de ser una anécdota, y el hecho de que esta circunstancia no haya tenido lugar no invalida la tesis de un conocimiento temprano de la pintura nórdica en el ámbito valenciano, porque a buen seguro Garcia conocería bien la tabla que se le iba a ceder, y Reixac habría visto el dibujo que custodiaba Garcia.

Es oportuno aquí recordar gusto del monarca por la pintura eyckiana ${ }^{27}$ : además del San Jorge al que se acaba de hacer referencia, Bartolomeo Fazio en De viris illustribus (1456) señala que entre las posesiones partenopeas del Rey se contaban un tríptico regalado o vendido por la familia Lomellini (probablemente su obra preferida, según Giovanni Pontano) ${ }^{28}$, y una "Adoración de los Magos". Las tres obras se han perdido, aunque su eco parece haber quedado en otras piezas que tal vez se inspiraron en ellas. Más que rastrear la huella de los originales perdidos en tablas conservadas de mayor o menor calidad, lo que interesa aquí es volver a subrayar la fama que debía tener entre las personas próximas a la Corte "lo gren pintor del illustre duch de Burgunya" 29 , tan del agrado de su Señor, y que sirve para explicar el valor que se pudo atribuir a obras que se consideraron salidas de su mano, fueran auténticas o no.

Sobre la cuestión de la autografía de las dos pinturas documentadas en Valencia a mediados del siglo XV como "de mà de Johannes", la que tiene más visos de haber salido de Brujas, como fácilmente se puede suponer, es la tabla de roble que viajó rumbo a Nápoles. Además de estar documentada y descrita repetidas veces (Libro de Cuentas de la Bailía, Fazio, Summonte...), el San Jorge eyckiano dejó una notable impronta en la pintura de las riberas del Mediterráneo: Colantonio realizó varias réplicas (no conservadas), hay una miniatura en el Libro de Horas de Alfonso de Aragón

\footnotetext{
${ }^{22}$ Paviot, 1990: 86 y nota 24.

${ }^{23}$ Paviot, 1990: 86, nota 22.

${ }^{24}$ El nombre de la galera genovesa lo da Strelhke (1997: 69), si bien no ha sido posible aclarar cuál es su procedencia exacta.

${ }^{25}$ Sanchis Sivera, 1914: 114. Ames Lewis (2000: 276) apunta que la pintura llegó desde Brujas vía Barcelona en 1444.

${ }^{26}$ Cerveró Gomis, 1964: 93.

27 Vid. Strelhke, 1997: 68-71; Eduard Mira, "Del Mar del Norte al Mediterráneo...”, en Natale, 2001: 117-132.

${ }^{28}$ Strelhke, 1997: 69.

${ }^{29}$ Sanchis Sivera, 1914: 114.
}

Arch. esp. arte, LXXXIX, 353, ENERO-MARZO 2016, 1-14 ISSN: 0004-0428, eISSN: 1988-8511, doi: 10.3989/aearte.2016.01 
que parece derivar de la composición original ${ }^{30}$, y en el San Jorge de Pere Nisart es fácil ver la huella de los intereses de Van Eyck, pero no su aliento, ya que la copia parece limitarse a los rasgos más anecdóticos, que fueron justamente los que consignó Summonte en su descripción ${ }^{31}$. En lo que se refiere a la "Estigmatización de San Francisco", curiosamente, debe hacerse el recorrido inverso: existe una sola huella documental que se refiere a la posible filiación de la tabla, pero ha quedado una importante estela de derivaciones formales, además de dos obras que se consideran como piezas originales del pintor brujense: la de la Galería Sabauda y la del Museo de Filadelfia, casi idénticas salvo en las dimensiones, muy diferentes entre sí, hecho que resulta sorprendente y ha puesto a prueba la capacidad de interpretación de la historiografía ${ }^{32}$. Quizás, el ejemplo que mejor pueda relacionarse con la existencia de un modelo eyckiano en Valencia sea una tabla conservada en el Convento de las Capuchinas de Castellón, que Gómez Frechina atribuye al Maestro de la Porciúncula, activo en la segunda mitad del siglo $\mathrm{XV}^{33}$ (fig. 2). Esto sería una constatación de la huella que la obra que poseía Reixac pudo dejar en el medio pictórico local, pero no constituiría un argumento irrefutable a favor de la atribución de esta "Estigmatización" a Van Eyck, por más que sea posible ligar compositivamente la obra castellonense y las de Turín y Filadelfia. Se sabe que el pintor flamenco

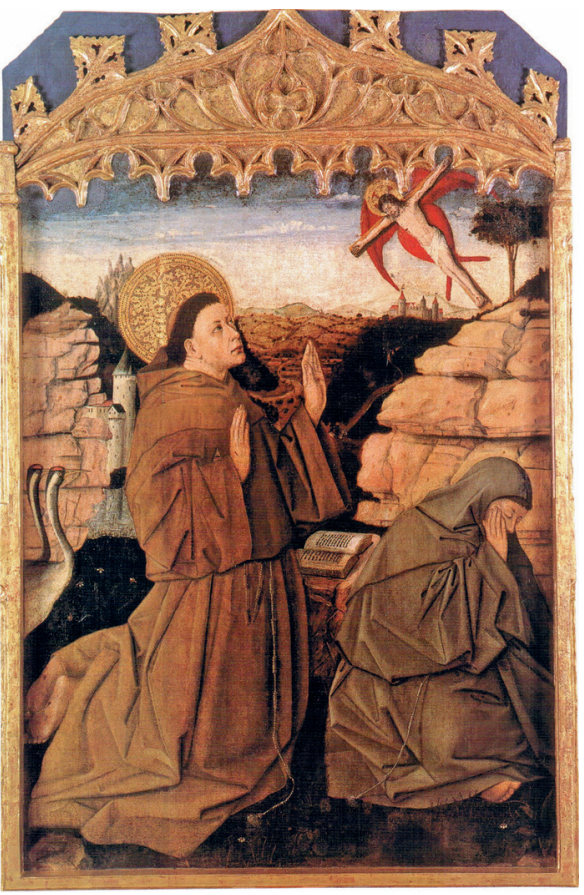

Fig. 2. Maestro de la Porciúncula, tabla de la "Estigmatización de San Francisco" procedente del Convento de Capuchinas de Castellón (ca. 1460). Museo de Bellas Artes de Castellón. llevó a cabo al menos dos trabajos con esa temática (los dos documentados y con dueño conocido, correspondan o no a los de la Galería Sabauda y el museo estadounidense), y que la tabla de Reixac estaba "acabada ab oli”, pero aun así es difícil aseverar que se tratara de una tercera versión autógrafa. La noción de copia fiel era muy familiar a la pintura flamenca, el mismo Colantonio cultivaba este arte de la réplica de manera excelente ${ }^{34}$, y se conoce

${ }^{30}$ Strehlke, 1997: 64 y fig. 42. Concomitancia también recordada por Toscano, 2001: 88 [el autor remite a Ferdinando Bologna, el primero en identificar el eco de la composición eyckiana: Bologna, Ferdinando. Napoli e le rotte mediterranee della pittura da Alfonso el Magnanimo a Ferdinando il Cattolico. Nápoles (Società Napoletana di Storia Patria), 1977, pp. 87-89].

${ }^{31}$ Una transcripción de la carta que Summonte remite a Marcantonio Michiel (1524), en Nicolini, 1923: 126. Hay que apuntar también que, aunque Summonte no atribuya la obra a Van Eyck, tanto la procedencia como la fama y las medidas de la tabla concuerdan con la pieza embarcada en Valencia.

${ }^{32}$ Para un estudio exhaustivo del asunto, vid. Rishel, 1997: 45-53, y, sobre todo, Katherine Crawford Luber. "San Francisco recibiendo los estigmas" [ficha de catálogo nº1], en Benito Doménech y Gómez Frechina, 2000: 106-117.

33 José Gómez Frechina: "San Francisco recibiendo los estigmas" [ficha de catálogo no 2], en Benito Doménech y Gómez Frechina, 2000: 118-123. Cfr. una reseña de la exposición en la que se hace particular hincapié en la tabla, en Strelhke, 2001: 590-593, esp. 590-591. Vid. también, más recientemente, Cornudella, 2008: 83-111, y esp. 94-95.

${ }^{34}$ Summonte escribió: "Fo in costui una gran dextrezza in imitar quel che volea; la qual imitazione ipso avea tutta convertita in le cose di Fiandra, che allora sole erano in prezzo" [transcripción en Nicolini, 1923: 125]; y, sobre el "San Jorge': "Insomma lo bon Colantonio la contrafece tutta questa pittura, di modo che non si discernea la sua da l'archetipo se non da un albero, che in quella era di róvola e in questa costui ad bel studio la volse fare di castagno" [transcripción en Nicolini, 1923: 126].

Arch. esp. arte, LXXXIX, 353, ENERO-MARZO 2016, 1-14

ISSN: 0004-0428, eISSN: 1988-8511, doi: 10.3989/aearte.2016.01 
bien la maestría con la que los talleres neerlandeses practicaron esa vertiente de la pintura ${ }^{35}$. Reflexionar sobre esto tiene implicaciones también para el caso que se está tratando, pudiendo ayudar a cercar el problema de la atribución del dibujo de Berenguer Mateu, porque trascendiendo otra vez la cuestión de su autenticidad, que se considerará a continuación, las obras que se citan como "de mà de Johannes" tienen con seguridad, al menos, el valor de un espejo que captó de modo exacto un particular y distinto modo de pintar y retuvo su reflejo, contemplado por los contemporáneos como algo verdaderamente nuevo, hubiera salido del taller del famoso artífice o no. Sobre la cuestión de la autoría de la "Estigmatización" se pronuncian Gómez Frechina ${ }^{36}$, Benito Doménech ${ }^{37}$ y Ximo Company $^{38}$ al atribuirla sin duda a Van Eyck, sustentando esta afirmación en la presencia del "San Jorge" en Valencia cuatro años antes de la redacción del testamento de Reixac, en la aparición de la obra del Maestro de la Porciúncula, en la preocupación manifiesta que muestra el testador por la suerte de la tabla, en el eco flamenco que se puede apreciar en la pintura valenciana de la segunda mitad de la centuria y en el dato preciso de que la pieza estaba pintada al óleo.

Tras haber examinado cuáles son los datos en los que se funda el paso de Van Eyck por Valencia y cuál fue la presencia de sus obras en la ciudad, procede ahora delimitar la huella de su arte en la pintura valenciana de la época ${ }^{39}$, tan diferente a la napolitana pese a estar igualmente expuesta a la influencia del pintor flamenco, con el objeto último de explorar si tiene visos de realidad la mención de un dibujo a pluma de su autoría en el inventario de los bienes de Andreu Garcia. En el reino partenopeo la llegada de las tablas eyckianas tuvo un impacto inmediato y duradero a través de Colantonio y de la figura de Antonello da Messina. Por contra, hasta hace poco en Valencia no se consideraba que hubiese nada de factura propiamente eyckiana después de la vuelta de Lluís Dalmau en 1436 con un repertorio de modelos flamencos que puede decirse en ignición, utilizados entre 1443 y 1445 en su "Verge dels Consellers" ${ }^{40}$. Del trabajo de Dalmau en el sexenio que va de 1436 a 1443 nada ha quedado, y muy poco se sabe. El "San Baldirio" de la iglesia de Sant Boi de Llobregat, fechado en 1448 , muestra ya un distanciamiento claro respecto a los modelos que el pintor tenía bien presentes sólo tres o cuatro años antes ${ }^{41}$. Lo que siguió fructificando espléndidamente en Nápoles parecía no

${ }^{35}$ Hasta el punto de formar con su habilidad un verdadero laberinto de tablas de roble en las que el estudioso tiende a perderse, si no fuera por los trabajos sobre el dibujo subyacente que se han llevado a término en Lovaina, principalmente, y que han confirmado la importante función de los dibujos y plantillas en el Norte. Vid. al respecto los estudios centrados específicamente en la pintura flamenca de Comblen-Sonkes, 1979: 44-45, lám. 2 y 3; Dijkstra, 1989: 37-53, lám. 8-13; Périer-D’Ieteren, 1979: 46-48, lám. 4 y 5; Périer-D’Ieteren, 1982-83: 74-94; Périer-D’Ieteren, 1985: 61-69, lám. 22-27. Para el ámbito hispánico, vid. las investigaciones pioneras de María del Carmen Garrido sobre pintura hispanoflamenca, publicadas en alguno de los coloquios de Lovaina (se citarán por extenso más adelante), y expuestas en una versión reciente en Finaldi y Garrido, 2006. Cfr. también Garrido y Bertani, 2010.

${ }^{36}$ Gómez Frechina, "San Francisco recibiendo los estigmas"..., en Benito Doménech y Gómez Frechina, 2000: 118120. Vid. también Gómez Frechina, "Algunas pautas flamencas en la pintura valenciana del siglo XV”, en Benito Doménech y Gómez Frechina, 2000: 63-103 (dentro de ese texto, "Claves Eyckianas en torno a la iconografía de la estigmatización de San Francisco", pp. 63-76).

${ }^{37}$ Fernando Benito Doménech, "Evocaciones flamencas en los Primitivos Valencianos", en Benito Doménech y Gómez Frechina, 2000: 23-62, especialmente pp. 23-26, aunque más ampliamente, hasta la página 45.

${ }^{38}$ Company, 1990: 30.

${ }^{39}$ Para una brevísima panorámica de la influencia de Van Eyck en la pintura hispana, vid. Bermejo, 1980: tomo I, 78; y Bermejo, 1990. En lo que se refiere específicamente a la Corona de Castilla, vid. Silva, 2002; y en lo que respecta a Aragón, vid. Yarza, 2002. Francesc Ruiz Quesada (Ruiz, 2007) revisa la cuestión de la influencia del realismo flamenco en Cataluña; y Rafael Cornudella (Cornudella, 2008) hace lo mismo para Valencia, a partir de la obra del Maestro de la Porciúncula. Joan Molina (Molina, 2003) se ocupa de los dos territorios.

${ }^{40}$ Company, 1990: 11-14. Respecto a la figura de Lluís Dalmau, vid. Tramoyeres, 1907; y mucho más recientemente, la revisión exhaustiva de Ruiz, 2007 (sobre la "Verge dels Consellers", vid. específicamente pp. 248-256). Una visión de contexto, en Jones, 2000: 198 y nota 10.

${ }^{41}$ Cfr. Ruiz, 2007: 257-262. 
poder sobrevivir sin aclimatarse en Barcelona o Valencia, a pesar de que sí se tenía cierto conocimiento de la pintura del Norte con anterioridad a la eclosión eyckiana ${ }^{42}$, y se conocía con seguridad la personalidad del eximio pintor de Brujas a través de los encargos que Alfonso V hizo a sus agentes valencianos. No obstante, un estudio reciente de Rafael Cornudella i Carré que se ocupa de forma concienzuda de la figura del Maestro de la Porciúncula, poniendo de relieve su sorprendente contacto con modelos de Van Eyck, describe la pintura valenciana de mediados del siglo XV, en lo que a esta cuestión concreta se refiere, como "un territorio extraordinario, que en ningún caso se circunscribe al célebre Dalmau, y que ofrece virtualidades y sorpresas no agotadas" ${ }^{\prime 43}, \mathrm{y}$, tras argumentar la idea con solvencia, alude al "amplio abanico de modelos eyckianos en manos de los pintores valencianos" ${ }^{\text {" }}$. Aun así, se hace necesario entender que, si bien Valencia fue la ciudad a la que arribó Lluís Dalmau después de su estancia en Flandes ${ }^{45}$, y acogió también el trabajo de Luis Alimbrot (Allynckbrood en la documentación belga $)^{46}$, se hizo preciso en algún caso reformular las aportaciones septentrionales para acomodarlas al gusto de la clientela de la urbe. Fueron Jacomart ${ }^{47}$ y Joan Reixac ${ }^{48}$ los encargados de esta adaptación. En la pintura de ambos es posible advertir una clara veta del ars nova de Flandes, si bien distanciada de referencias propiamente eyckianas. En este momento sería interesante señalar una noticia que podría servir como punto de partida para un nuevo examen de las fuentes artísticas que ambos pudieron tener a su disposición. Gonçal Sarrià, Garcia Sarrià (sobrino suyo) y Joan Reixac tasaron en doscientos veinte sueldos una obra de Lluís Dalmau en $1439^{49}$, un San Miguel para la clave de madera de una tienda del Rey. El hecho resulta muy significativo, por cuanto muestra a dos artífices bien conocidos estimando el valor de una pieza que a la historiografía le hubiera gustado ver: un trabajo del recién regresado Dalmau, pleno, cabe suponer, de alusiones a la obra de Van Eyck. Como se acaba de apuntar, cuando se reflexiona sobre Jacomart y Reixac se suele subrayar, sobre todo, su personal interpretación de los aportes flamencos. Sin embargo, no estuvieron apartados de los modelos eyckianos, sino muy próximos a ellos: Jacomart a través de sus dos viajes a Nápoles, cuando podría contemplar las obras del maestro brujense que estaban en poder del monarca, y Reixac a través de su misma "Estigmatización", del contacto con Andreu Garcia y su valioso depósito de dibujos, y de la tasación de la obra de Dalmau. Cabe apuntar, por otra parte, que la figura de San Antonio Abad en el retablo de los Martí de Torres que se conserva en el Museo de Bellas Artes de Valencia, atribuido a Gonçal Peris Sarrià, sí muestra algo de la imponente presencia de las figuras eyckianas. No debe olvidarse, en este punto, que Sarrià trabajó con Jaume Mateu, hermano de Berenguer, entre 1427 y 1428 , y quizás después ${ }^{50}$, tasó un trabajo de Dalmau en 1438, eligió como testigo de su testamento a Reixac en $1451^{51}$, y mantuvo estrechos lazos con Andreu Garcia ${ }^{52}$, lo que supondría la posibilidad de un co-

${ }^{42}$ Joan Aliaga, en "El arcángel Gabriel; La Virgen anunciada" [ficha de catálogo nº 10, en Natale, 2001: 188], aporta un dato revelador, exhumado por Lluïsa Tolosa y Maite Framis: en 1425 un notario de Valencia, Bernat Gallach, es poseedor de "unes taules de roure de Flandes en les quals és la passió de Ihesuchrist e de la resurrecció e apparició que feu Ihesuchrist a sent Thomàs".

${ }^{43}$ Cornudella, 2008: 94.

${ }^{44}$ Cornudella, 2008: 100. El autor indaga sobre las posibles vías de llegada de estos modelos en la página102.

${ }^{45}$ Company, 1990: 9-10.

46 Vid. Company, 1990: 14-15; Pilar Silva Maroto. "Tríptico de la Crucifixión” [ficha de catálogo n 36], en Natale, 2001: 288-290; José Gómez Frechina. "Natividad" [ficha de catálogo n 17], en Benito Doménech y Gómez Frechina, 2000: 168-171, le atribuye un pequeño Nacimiento de una colección privada.

47 Vid. Company, 1990: 15-27; Natale, 2001: fichas de catálogo no 46, 47, 48 y 49; Benito Doménech y Gómez Frechina, 2000: fichas de catálogo no 22 y 23; Ferre i Puerto, 2000: 1681-1686.

48 Vid. Company, 1990: 27-42; NATALE, 2001: fichas de catálogo nº 47, 48 y 49; Benito Doménech y Gómez Frechina, 2000: fichas de catálogo ${ }^{\circ}$ 6, 7, 24, 25-42, 44, 45, 48 y 49; y, sobre todo, los estudios de Josep Ferre i Puerto, especialmente Ferre, 1999: 419-426.

49 Aliaga Morell, 1996: doc. 67

${ }^{50}$ Cfr. Aliaga Morell, 1996: 99-100, docs. 42, 43; 68, doc. 83

${ }^{51}$ Vid. Company, 1990: 30; Aliaga Morell, 1996: 68 y doc. 83.

Arch. esp. arte, LXXXIX, 353, ENERO-MARZO 2016, 1-14

ISSN: 0004-0428, eISSN: 1988-8511, doi: 10.3989/aearte.2016.01 
nocimiento próximo de los modelos que obraban en poder de los cuatro. Se puede seguir sosteniendo con fundamento, entonces, junto a Carl Brandon Strehlke, que "L'arte di van Eyck, e quella fiamminga in genere, può essere intesa come il collante che ha unito le diverse tradizioni pittoriche di alcune città portuali del Mediterraneo come Valencia, Barcellona, Napoli o Genova". ${ }^{53}$ Un dibujo de Eyck, autógrafo o no, podría ser reconocido y valorado en Valencia a mediados del siglo XV.

Por fin es tiempo de abordar la cuestión de la autenticidad de la obra reseñada en el inventario de Andreu Garcia. Dos reflexiones se hacen necesarias: la primera, sobre el valor de la obra autógrafa en el tiempo y en el lugar de los que ha emergido el documento; la segunda, sobre las posibilidades que podría tener este dibujo a pluma de encajar en la producción de Van Eyck. Para empezar, aquí se va a asociar el valor de la obra autógrafa en Valencia entre fines del siglo XIV y mediados del XV a la expresión "de mà de", que es la que se utiliza en el registro. Ha sido posible localizar tres ejemplos. El primero de ellos está ligado al recuerdo de un insigne teólogo, y no guarda relación alguna con una empresa artística: en 1416, en el inventario de los bienes de Joan Mateu, presbítero, párroco de El Cuervo, y beneficiado en la Seu, se encuentra "un quernet de paper scrit de la mà de mestre Francesch Eiximenis" "54. Más interés para lo que aquí se está tratando de dilucidar es la mención de un libro "storiat de mà d'en Ferrer Bassa, pintor" que se utiliza como prenda en un préstamo de 10 florines en $1393^{55}$. En tercer y último lugar, el testimonio que cronológica y culturalmente más se aproxima a la alusión al dibujo de Garcia es el contrato, en 1441, del bordado de la orla del paño funerario que cubrirá los cuerpos de los cofrades difuntos de la Cofradía de Santa María de la Catedral. En las capitulaciones, se especifica que se deberá llevar a término la labor "segons la mostra la qual han liurada als dits brodadors pintada de mà d'en Johan Rexach pintor" ${ }^{\prime \prime 5}$. En el caso de Eiximenis, la expresión "de mà de" significa con mucha probabilidad autografía (es difícil interpretar de otro modo la noticia). En lo que se refiere a los ejemplos vinculados con Ferrer Bassa y Joan Reixac, también debe querer significar lo mismo, esto es: el dueño del libro y los contratantes del bordado saben que las miniaturas y la mostra salieron del taller de un determinado artífice.

Corresponde ahora revisar los pocos datos que la documentación proporciona sobre el diseño perteneciente en origen a Berenguer Mateu con el objeto de comprobar si no se contradicen con lo que se sabe respecto a los dibujos de Jan van Eyck ${ }^{57}$. La pieza que Mateu dejó a Garcia en depósito era una media figura en papel, trazada a pluma. El único dibujo original de Van Eyck que se conserva es el retrato del cardenal Niccolò Albergati, conservado en Dresde (el único dibujo flamenco, en realidad, que se puede atribuir a un artista individual con seguridad antes de El Bosco, y esto ya es bastante significativo sobre la noción de original y copia en el Norte). En este caso, la técnica utilizada es la punta de plata, y el encuadre escogido es el del busto, como en muchos otros retratos que pueden atribuirse a Eyck o a su círculo más próximo, asimilable a la "mitja ymatge" de la documentación valenciana. Puede suponerse también el detalle con el que debería estar terminada la obra: en primer lugar, porque la minuciosidad en el acabado era un rasgo inequívocamente propio de la pintura flamenca; en segundo lugar, porque, comparando el retrato de Albergati sobre papel y sobre tabla, ha sido posible concluir que el artista concedió al diseño previo una importancia notable ${ }^{58}$. Por otra parte, la pluma fue una herramienta habitual de trabajo entre los pintores neerlandeses, junto con la plumilla de metal y el pincel. Así, tanto el formato como la técnica del ejemplar de Berenguer Mateu parecen concordar con prácticas bastante usuales en los talleres septentrionales.

\footnotetext{
${ }^{52}$ Lo eligió como albacea de su testamento (Aliaga Morell, 1996: 68 y doc. 83).

${ }^{53}$ Strehlke, 1997: 68.

${ }^{54}$ APCCV, no 6419, Domènec Barreda, 1416 (12 de octubre).

${ }_{55}$ Company, Aliaga, Tolosa y Framis, 2005: doc. 640, p.364.

${ }^{56}$ Archivo del Reino de Valencia (ARV), Protocolos, $\mathrm{n}^{\circ}$ 2411, Vicent Çaera, 1441 (12 de abril), p. 249v (numerado

${ }^{57}$ La cantidad de copias es altísima, e inversamente proporcional a los originales conservados (Buck, 2000: 183-195).

${ }^{58}$ Buck, 2000: 184.
} en lápiz). 
El último aspecto que va a revisarse aquí del dibujo citado en el inventario valenciano es su valor económico. Se sabe que la media figura "de mà de Johannes", un libro de pintura y cuatro tablillas con cabezas pintadas son una fianza de Berenguer Mateu, pintor, hermano de Jaume Mateu, por un préstamo de seis florines. A Garcia en 1452 ya le había sido devuelta la mitad de esta cantidad y, a juzgar por la nota que informa sobre la procedencia de los objetos, el depósito seguía en sus manos. El presbítero estipula en su manuscrito que es su voluntad que el libro sea devuelto a su hermano o a los suyos, a quien pertenece. Esto implicaría, además de la defunción de Berenguer Mateu, que el dibujo y las tablillas se estiman en tres florines, y el libro de pintura en otros tres, por cuanto se manda devolver sólo el volumen, y no los diseños. Resulta cuanto menos curioso el alto precio en el que se habría tasado el primero, y permanecería incierto el paradero de los segundos. En cualquier caso, trabajando sobre la hipótesis de una tasación de la "mitja ymatge" que oscilaría entre un florín y medio o dos, se encuentra que era un precio estimable para un dibujo, poniéndolo en comparación con otras cantidades pagadas a figuras destacadas de la pintura de aquellos años: por ejemplo, a Gonçal Peris Sarrià se le pagan parte de 125 libras en 1441 por un retablo para la iglesia de San Martín de Valencia ${ }^{59}$, y 50 florines en 1444 por un retablo para la iglesia de san Miguel de Murcia ${ }^{60}$; Reixac recibiría 36 libras por un retablo que contrata en 1456 para la testamentaría de Joan Bellmunt ${ }^{61}$. A pesar de que sería posible aportar el coste de muchos más trabajos de pintura contratados en Valencia a mediados del siglo XV, el aluvión de cantidades resultaría del todo irrelevante sin un análisis pormenorizado de las circunstancias de cada acuerdo, puesto que "el precio no debe ser asumido acríticamente como indicación, fuera de pactos y de contexto social" $"$.

En conclusión, los datos analizados conducen a pensar que la presencia en el domicilio del presbítero Andreu Garcia de un dibujo de raíz eyckiana es verosímil: aunque "Johannes" efectivamente no formara parte de la embajada borgoñona que estuvo en Valencia en el otoño de 1427, en la ciudad sí se conocía y admiraba su pintura, y no sólo por parte del Rey y su círculo cortesano, sino incluso en su mismo medio profesional (Berenguer Mateu y Joan Reixac poseían obras atribuidas a su mano). La autografía es otra cuestión. Si el dibujo es auténticamente obra de Van Eyck o no es muy difícil saberlo, y todavía más arriesgado suponerlo. Aun así, hay un hecho clave: tanto la "Estigmatización" de Reixac como la "mitja ymatge" de Mateu eran piezas relacionables con la producción del pintor brujense. Debían, por tanto, tener rasgos característicos, nuevos, distintos, flamenquizantes. Y más aún: los modelos eyckianos no sólo estaban en poder de un grupo selecto de comitentes como el Rey o los presbíteros relacionados con la Catedral y sus empresas artísticas, o de Reixac, el más prolífico de los pintores valencianos del momento, sino también de Berenguer Mateu, miembro de una saga clave en la segunda generación del Gótico Internacional en Valencia.

Tras esto, es necesario relacionar el depósito de Berenguer Mateu con otros objetos parecidos (tablillas dibujadas, muestras) que se registran en el inventario del presbítero. Puede aventurarse que estos enseres podrían constituir también la garantía de un préstamo a algún artífice. A través del inventario de sus bienes, se sabe que Andreu Garcia no sólo dejó dinero a Mateu o a Gonçal Sarrià, sino también a Joan Reixac y al iluminador Pere Bonora ${ }^{63}$. Además, disponía de varios inmuebles que alquiló a profesionales relacionados con la producción artística, y probablemente vendió censos a algunos de ellos. Por contra, no hay nota alguna de Garcia que especifique que la posesión de estos artículos derivara de sus gestiones financieras, orientadas de modo más que evidente hacia el préstamo de pequeñas cantidades, fuera esto o no un negocio lucrativo. De todos modos, no deja de ser factible una hipótesis muy atractiva, que es la de la formación, en vida del finado,

\footnotetext{
59 Aliaga Morell, 1996: doc. 79.

${ }^{60}$ Aliaga Morell, 1996: doc. 81.

${ }^{61}$ Company, 1990: 31-33.

${ }^{62}$ Intervención de Paulino Iradiel Murugarren en el coloquio sobre Mercats del luxe, mercats de l'art. La Corona d'Aragó i la Mediterrània en els segles XIV i XV [7/IX/2010].

${ }_{63}$ APCCV, Ambròs Alegret, no 1107 (diciembre de 1452, en un cuadernillo de pagos independiente).
}

Arch. esp. arte, LXXXIX, 353, ENERO-MARZO 2016, 1-14

ISSN: 0004-0428, eISSN: 1988-8511, doi: 10.3989/aearte.2016.01 
de una suerte de códice facticio, disperso en su domicilio, que reunía las herencias figurativas más importantes de la segunda remesa de artistas del estilo 1400 en Valencia (Sarrià, los Mateu, y Pere Bonora), y de la primera de la nueva pintura de influencia flamenca (Jacomart y Reixac) a través de la compra que pudo hacer el eclesiástico de los objetos que le resultaran más apetecibles, puesto que lo tenía al alcance, a través de sus relaciones y de las eventuales necesidades económicas de sus protégés. La figura de Garcia, así, bien podría contribuir a despejar alguna incógnita en la ecuación de varios grados que es la pintura valenciana en los años 30-50 del siglo XV.

Entre 1430 y 1452, y por aludir sólo a datos relacionados directamente con la cuestión que se está examinando en este artículo, Berenguer Mateu pinta el retablo de Jérica (ca.1431), Lluís Dalmau vuelve a Valencia tras su viaje a Flandes (1436), Jaume Huguet trabaja junto a Jaume Mateu en la ciudad (1445) $)^{64}$, Joan Reixac cita en su testamento la tabla de la "Estigmatización de san Francisco" (1448), muere Berenguer Mateu (1449), testa Andreu Garcia (1450), y en el inventario de sus bienes se cita la "myga imatge en paper, de ploma, de mà de Johannes" (1452). Justo entonces, a mediados del siglo XV, el todavía anónimo Maestro de la Porciúncula está en activo desplegando un sorprendente repertorio de modelos eyckianos en las tablas que se le atribuyen. Falta determinar los medios específicos que sirvieron para verter iconografías y formas foráneas al lenguaje artístico local. El tránsito de dibujos pudo ser una vía adecuada de transmisión de innovaciones, aunque no sirve para solucionar la frecuente discordancia entre las novedades que significaban los modelos importados y la pintura practicada por sus dueños. Es éste el caso de los hermanos Mateu, en contacto con al menos una obra atribuida a la mano de Van Eyck (si no autógrafa, inequívocamente flamenca), y ejecutores de una pintura que en el mejor de los casos se ha calificado de retardataria o, al menos, claramente inferior a la practicada por su tío Pere Nicolau. Sin embargo, es Berenguer quien poseyó el dibujo a pluma que se ha venido estudiando, y es con Jaume con quien colabora Jaume Huguet en una fase temprana de su carrera. Sin desestimar que estos hechos se debieran a cuestiones más bien fortuitas, o a asuntos más relacionados con la estrategia profesional que con la excelencia artística -algo que podría explicar la incursión de Huguet en el mercado del extremo meridional de la Corona de Aragón-, las figuras de los Mateu constituyen un ejemplo no único, pero sí singular, que obliga a revisar de nuevo cualquier teoría historiográfica que dé por supuesta una adhesión inmediata, irreversible y acrítica a modelos foráneos ${ }^{65}$, en razón de su prestigio y disponibilidad, sin atender a otra circunstancia.

\section{BIBLIOGRAFÍA}

Aliaga Morell, Joan (1996): Els Peris i la pintura valenciana medieval. Valencia, Edicions Alfons el Magnànim.

Aliaga Morell, Joan / Rusconi, Stefania (en prensa): "Nuevas aportaciones a la pintura del gótico internacional. Berenguer Mateu y el retablo de Jérica (Castellón)".

Ames-Lewis, Francis (2000): The Intellectual Life of the Early Renaissance Artist. New Haven-Londres, Yale University Press.

Benito Doménech, Fernando / Gómez Frechina, José (dirs.) (2001): La Clave Flamenca en los Primitivos Valencianos [catálogo]. Valencia (Museo de Bellas Artes de Valencia).

Bermejo Martínez, Elisa (1980): La pintura de los primitivos flamencos en España. Madrid, CSIC-Instituto 'Diego Velázquez', tomo I.

Bermejo, Elisa (1990): "Influencia de Van Eyck en la pintura española". En: Archivo Español de Arte, no 252, pp. 555-569.

\footnotetext{
${ }^{64}$ Ferre, 2003.

${ }^{65}$ Un perspicaz estudio de la recepción y de la fortuna crítica de la pintura flamenca en Italia (algo difícilmente viable para la península Ibérica, dada la escasez de literatura artística anterior al siglo XVI), en Christiansen, 1998.
}

Arch. esp. arte, LXXXIX, 353, ENERO-MARZO 2016, 1-14 ISSN: 0004-0428, eISSN: 1988-8511, doi: 10.3989/aearte.2016.01 
Blackman, Christabel / Ferre Puerto, Josep A. (2001): "Retaule de Sant Jordi de Jérica”. En: Recuperem patrimoni, $\mathrm{n}^{\mathrm{o}} 4$.

Buck, Stephanie (2000): "An Approach to Looking at Eyckian Drawings". En: Foister, S. / Jones, S. / Cool, D. (eds.): Investigating Jan van Eyck. Turnhout, Brepols, pp. 183-195.

Cerveró Gomis, Luis $(1963,1964)$ : “Pintores valentinos. Su cronología y documentación”. En: Anales del Centro de Cultura Valenciana [Segunda Época], 48, pp. 63-156; y 49, pp. 83-136.

Cerveró Gomis, Luis $(1966,1971)$ : "Pintores valentinos. Su cronología y documentación”. En: Archivo de Arte Valenciano, año XXXVII, pp. 19-30; y año XLII, pp. 23-36.

Christiansen, Keith (1998): "The View from Italy". En: Ainsworth, M.W. / Christiansen, K. (eds.): From Van Eyck to Brueghel. Early Netherlandish Painting in the Metropolitan Museum of Art [catálogo de exposición]. Nueva York, Metropolitan Museum of Art, pp. 39-61.

Comblen-Sonkes, M. (1979): "Le dessin mecanique chez les primitifs flamands". En: Hollanders-Favart, D. / Van Schoute, R. (eds.): Le dessin sous-jacent dans la peinture. Lovaina, Université Catholique de Louvain, pp. 44-45, lám. 2 y 3.

Company i Climent, Ximo (1990): La pintura hispanoflamenca. Valencia, Alfons el Magnànim.

Company i Climent, Ximo (1991): La pintura dels Osona: una crü̈lla d'hispanismes, flamenquismes i italianismes. Lleida, Pagès, 2 vols.

Company, Ximo / Aliaga, Joan / Tolosa, Lluïsa / Framis, Maite (2005): Documents de la pintura valenciana medieval i moderna, I (1238-1400). Valencia, Universitat de València.

Cornudella, Rafael (2008): "El Mestre de la Porciúncula i la pintura valenciana del seu temps". En: Butlletí $M N A C$, , pp. 83-111.

Cornudella, Rafael (2009-2010): “Alfonso el Magnánimo y Jan van Eyck. Pintura y tapices flamencos en la corte del rey de Aragón”. En: Locus Amoenus, n 10, pp. 39-62.

Dijkstra, Jellie (1989): "On the Role of Underdrawings and Modeldrawings in the Workshop Production of the Master of Flémalle and Rogier van der Weyden". En: Verougstraete, H. / Van Schoute, R. (eds.): Géographie et chronologie du dessin sous-jacent. Lovaina, Université Catholique de Louvain, pp. 37-53, lám. 8-13.

Ferre i Puerto, Josep (2000): "Jacomart, lo feel pintor d'Alfons el Magnànim: Puntualitzacions a l'obra valenciana". En: VVAA. XVI Congresso Internazionale di Storia della Corona d'Aragona. La Corona d'Aragona ai tempi di Alfonso il Magnanimo. Napoli-Caserta-Ischia. 18-24 setembre 1997. Nápoles, Paparo Edizioni, pp. 1681-1686.

Ferre i Puerto, Josep (1999): "Trajectòria vital de Joan Reixac, pintor valencià del quatre-cents: La seua relació amb Andreu Garcia”. En Yarza, J. / Fité, F. (eds.): L'artista-artesà medieval a la Corona d'Aragó. Lleida, Universitat de Lleida, Institut d'Estudis Ilerdencs, pp. 419-426.

Ferre i Puerto, Josep (2003): "Presència de Jaume Huguet a València. Novetats sobre la formació artística del pintor". En: Ars Longa, n $^{\circ}$ 12, pp. 27-32.

Finaldi, Gabriele / Garrido, Carmen (eds.) (2006): El trazo oculto. Dibujos subyacentes en pinturas de los siglos XV y XVI [El Prado, del 20 de julio al 5 de noviembre de 2006]. Madrid, Museo Nacional del Prado.

Foister, Susan / Jones, Sue / Cool, Delphine (eds.) (2000): Investigating Jan van Eyck. Turnhout (Brepols).

Fransen, Bart (2012): "Jan van Eyck y España. Un viaje y una obra”. En: Anales de Historia del Arte, n 22, pp. 39-58.

Garrido, Carmen / Bertani, Duilio (comisarios) (2010): El nacimiento de una pintura: de lo visible a lo invisible [Museo de Bellas Artes de Valencia, julio-octubre 2010]. Valencia, Generalitat Valenciana.

Harbison, Craig (1991): "The Artist's Place at the Burgundian Court". En: Harbison, Craig: Jan van Eyck. The Play of Realism. Londres, Reaktion Books, pp. 19-24.

Jones, Sue (2000): “The Use of Patterns by Jan van Eyck's Assistants and Followers". En: Foister, S. / Jones, S. / Cool, D. (eds.): Investigating Jan van Eyck. Turnhout, Brepols, pp. 197-207.

Llanes i Domingo, Carme (2011): L'obrador de Pere Nicolau i la segona generació de pintors del gòtic internacional a València [tesis doctoral leída en la Universitat de València en septiembre].

Molina Figueras, Joan (2003): "Ecos de la pintura flamenca en Valencia y Cataluña". En: Carbonell, E. / Casanelli, R. (eds.): El Mediterráneo y el Arte. Del Gótico al inicio del Renacimiento. Barcelona, Lunwerg, pp. 217-229.

Montero Tortajada, Encarna (2007): "Recetarios y papers de pintura en la documentación bajomedieval. Valencia, 1452: el ejemplo de Andreu García". En Libros con arte, arte con libros. Extremadura, Universidad de Extremadura-Consejería de Cultura y Turismo, pp. 507-517.

Arch. esp. arte, LXXXIX, 353, ENERO-MARZO 2016, 1-14

ISSN: 0004-0428, eISSN: 1988-8511, doi: 10.3989/aearte.2016.01 
Montero Tortajada, Encarna (2013): La transmisión del conocimiento en los oficios artísticos. Valencia, 1370-1450 [tesis doctoral leída en la Universitat de València en septiembre].

Montero Tortajada, Encarna (2013): "El oligarca y los pinceles: breve semblanza del presbítero Andreu Garcia". Espacio, Tiempo y Forma, serie VII, nº 1 .

Natale, M. (comisario) (2001): El Renacimiento Mediterráneo. Viajes de artistas e itinerarios de obras entre Italia, Francia y España en el siglo XV [catálogo]. Madrid, Fundación Thyssen-Bornemisza.

Nicolini, Fausto (1923): “Pietro Summonte, Marcantonio Michiel e l'arte napoletana del Rinascimento". En: Napoli Nobilissima, vol. III, pp. 121-146; 159-172.

Pächt, Otto (1994): Van Eyck and the Founders of Early Netherlandish Painting. Londres (Harvey Miller). Panofsky, Erwin (1998): Los primitivos flamencos. Madrid, Cátedra (1 $1^{\mathrm{a}}$ ed. en 1953).

Paviot, Jacques (1990): "La vie de Jan van Eyck selon les documents écrits". En: Revue des archéologues et historiens de l'art de Louvain, XXIII, pp. 83-93.

Pemán y Pemartín, César (1969): Juan van Eyck y España. Cádiz, Museo Provincial de Bellas Artes de Cádiz.

Périer-D'Ieteren, C. (1979): "Usage du poncif dans la peinture flamande des XVe et XVIe siècles". En: Hollanders-Favart, D. / Van Schoute, R. (eds.): Le dessin sous-jacent dans la peinture. Lovaina, Université Catholique de Louvain, pp. 46-48, lám. 4 y 5.

Périer-D'Ieteren, Catheline (1982-83): "Dessin au poncif et dessin perforé. Leur utilisation dans les anciens Pays-Bas au XVe siècle”. En: Bulletin de l'Institut Royal du Patrimoine Artistique, nº 19, pp. 74-94.

Périer-D'ieteren, Catheline (1985): "La technique du dessin sous-jacent des peintres flamands des Xve et XVIe siècles. Nouvelles hypothèses de travail”. En: Van Shoute, R. / Hollanders-Favart, D.: Le dessin sous-jacent dans la peinture. Lovaina, Université Catholique de Louvain, pp. 61-69, lám. 22-27.

Rishel, Joseph (1997): "I dipinti di Filadelfia e di Torino: letteratura e problemi di attribuzione". En: Rishel, J.J. / Spantigati, C. (comisarios): Jan van Eyck (1390 c.-1441). Opere a confronto [catálogo de exposición: Galería Sabauda, 3 de octubre - 14 de diciembre de 1997]. Turín, Umberto Allemandi \& Co, pp. 45-53.

Ruiz Quesada, Francesc (2007): “Lluís Dalmau y la influencia del realismo flamenco en Cataluña”. En: Lacarra, $\mathrm{M}^{\mathrm{a}} \mathrm{C}$. (ed.): La pintura gótica durante el siglo XV en tierras de Aragón y en otros territorios peninsulares. Zaragoza, Institución Fernando el Católico, pp. 243-297.

Sanchis Sivera, José (1914): Pintores medievales en Valencia. Barcelona, Tipografía L'Avenç [edición facsímil de Librerías París-Valencia].

Silva Maroto, Pilar (2002): "La couronne de Castille et la Flandre". En: Borchert, T.-H. (ed.): Le siècle de Van Eyck (1430-1530). Le monde méditerranéen et les primitifs flamands. Brujas, Ludion, pp. 143-151.

Silva Maroto, Pilar / Garrido, María del Carmen (1995): "Le processus créatif chez Pedro Berruguete". En: Verougstraete, H. / Van Schoute, R. (eds.): Le dessin sous-jacent dans le processus de création. Lovaina, Université Catholique de Louvain, pp. 31-36; lám. 11-17.

Sterling, Charles (1976): “Jan van Eyck avant 1432”. En: Revue de l'art, n³3, pp. 7-82.

Strehlke, Carl Brandon (1997): "Jan van Eyck: un artista per il Mediterraneo". En: Rishel, J. J. / Spantigati, C. (comisarios): Jan van Eyck (1390 c.-1441). Opere a confronto [catálogo de exposición: Galería Sabauda, 3 de octubre - 14 de diciembre de 1997]. Turín, Umberto Allemandi \& Co, pp. 55-76.

Strehlke, Carl Brandon (2001): "Valencia. Early Flemish and Valencian Art". En: Burlington Magazine, no 1182, vol. CXLIII (septiembre), pp. 590-593.

Toscano, Gennaro (2001): "Nápoles y el Mediterráneo. Relaciones entre miniatura y pintura en la transición de la casa de Anjou a la casa de Aragón”. En: Natale, M. (comisario): El Renacimiento Mediterráneo. Viajes de artistas e itinerarios de obras entre Italia, Francia y España en el siglo XV [catálogo]. Madrid, Fundación Thyssen-Bornemisza, pp. 79-99.

Tramoyeres Blasco, Luis (1907): "El pintor Luis Dalmau”. En: Cultura española, no 5/2, pp. 553-581.

Weale, W. H. James (1908): The Van Eycks and Their Art. Hubert and John van Eyck, Their Life and Work. Londres-Nueva York, John Lane.

Yarza Luaces, Joaquín (2002): "La couronne d'Aragon et la Flandre”. En: Borchert, T.-H. (ed.): Le siècle de Van Eyck (1430-1530). Le monde méditerranéen et les primitifs flamands. Brujas, Ludion, pp. 129-133.

Fecha de recepción: 02-VI-2014

Fecha de aceptación: 02-XII-2014

Arch. esp. arte, LXXXIX, 353, ENERO-MARZO 2016, 1-14 ISSN: 0004-0428, eISSN: 1988-8511, doi: 10.3989/aearte.2016.01 